\title{
Irradiation hardening of pure tungsten exposed to neutron irradiation*
}

\author{
Xunxiang Hu ${ }^{1}$, Takaaki Koyanagi ${ }^{1}$, Makoto Fukuda ${ }^{2}$, N.A.P. Kiran Kumar ${ }^{1}$, Lance. L. Snead ${ }^{3}$, Brian D. \\ Wirth $^{1,4}$, Yutai Katoh ${ }^{1}$ \\ ${ }^{1}$ Oak Ridge National Laboratory, Oak Ridge, TN 37831 USA \\ 2 Tohoku University, Aoba-ku, Sendai 980-8576 Japan \\ ${ }^{3}$ Massachusetts Institute of Technology, Cambridge, MA 02139 USA \\ ${ }^{4}$ University of Tennessee, Knoxville, TN 37996 USA
}

\begin{abstract}
Pure tungsten samples have been neutron irradiated in HFIR at $90 \sim 850^{\circ} \mathrm{C}$ to $0.03 \sim 2.2$ dpa. A dispersed barrier hardening model informed by the available microstructure data has been used to predict the hardness. Comparison of the model predictions and the measured Vickers hardness reveals the dominant hardening contribution at various irradiation conditions. For tungsten samples irradiated in HFIR, the results indicate that voids and dislocation loops contributed to the hardness increase in the low dose region (< $0.3 \mathrm{dpa}$ ), while the formation of intermetallic second phase precipitation, resulting from transmutation, dominates the radiation-induced strengthening beginning with a relatively modest dose (>0.6 dpa). The precipitate contribution is most pronounced for the HFIR irradiations, whereas the radiation-induced defect cluster microstructure can rationalize the entirety of the hardness increase observed in tungsten irradiated in the fast neutron spectrum of Joyo and the mixed neutron spectrum of JMTR.
\end{abstract}

\section{Introduction}

Given its high melting temperature, high thermal conductivity, low sputtering yield, and low tritium retention in un-irradiated conditions, tungsten has been chosen as the plasma facing material for International Thermonuclear Experimental Reactor (ITER), and planned for use in future Demonstration fusion nuclear power station (DEMO) and fusion reactors [1] [2]. The tungsten plasma-facing components (PFCs) of fusion reactors will experience an extreme environment characterized by high temperature, high thermal flux, as well as intense particle fluxes (i.e., high energy neutrons, hydrogen isotope ions, helium ions), and complex engineering structural design associated with heat removal, and the combined sum of these loading conditions will impose significant challenges to tungsten performance [3, 4] [5] [6]. In particular, the $14 \mathrm{MeV}$-peak neutron irradiation produces significant displacement damage of the lattice structure creating vacancies, interstitials, and their clusters, as well as generating significant concentrations of

\footnotetext{
* This manuscript has been authored by UT-Battelle, LLC under Contract No. DE-AC05-00OR22725 with the U.S. Department of Energy. The United States Government retains and the publisher, by accepting the article for publication, acknowledges that the United States Government retains a non-exclusive, paid-up, irrevocable, world-wide license to publish or reproduce the published form of this manuscript, or allow others to do so, for United States Government purposes. The Department of Energy will provide public access to these results of federally sponsored research in accordance with the DOE Public Access Plan (http://energy.gov/downloads/doe-public-access-plan).
} 
transmutation elements (i.e., Re, Os) [7]. Severe thermo-mechanical property degradation of tungsten is expected as a result of the irradiation-induced defect accumulation [8] [9, 10]. In turn, the degradation of these properties could impact plasma materials interactions (PMI), such as the bulk tritium retention, temperature-dependentmechanisms (e.g., chemical sputtering) impacted by the change of thermal conductivity.

The physical and mechanical properties of unirradiated, ITER grade tungsten are well understood as provided in the ITER Materials Properties Handbook [11]. However, there is very limited experimental data on the neutron irradiation response of tungsten. The research activities on neutron irradiation response of tungsten and its alloys conducted before the 1990s [12] [13] [14] were focused on the space application of liquid-metalcooled fast reactors in terms of the defect cluster evolution, including void and precipitation formation. More recently, the irradiation effects of tungsten have received a great deal of attention in the nuclear materials community worldwide [15] [16] [17] [9] [18] in response to its application in fusion reactors as plasma facing material. Oak Ridge National Laboratory (ORNL), USA is conducting a large neutron irradiation campaign to systematically investigate the resulting microstructure and thermo-mechanical property degradation of tungsten subject to neutron irradiation by closely collaborating with Japanese fusion community. A comprehensive paper summarizing the scientific findings from this campaign is being prepared by Dr. L. Snead and will be published soon [19]. The present work, as a part of this neutron irradiation campaign, is focused on irradiation hardening of tungsten.

Embrittlement is one of the most significant degradation phenomena of concern for tungsten exposed to irradiation at low to intermediate temperature, which is manifest as an increase in the ductile-to-brittle transition temperature (DBTT) that will impact the service lifetime and performance of tungsten PFCs. Hasegawa [9] [18] [20] and Fukuda [21] [10] [22] reported the neutron irradiation effects on the microstructural development of tungsten and tungsten alloys irradiated in various fission reactors, as well as the resulting hardening behavior by capturing the dependence of neutron energy spectra, irradiation temperature, and dose levels. Hu et al. [23] reported positron annihilation spectroscopy measurements and TEM observations on neutron-irradiated single crystalline tungsten irradiated in HFIR. The obtained microstructure information was then used to link to the measured hardness evolution during the isochronal annealing process. However, the underlying mechanisms governing the irradiation hardening behavior of neutron-irradiated tungsten has not been systematically reported in terms of the individual hardening contribution of different types of radiation defects present within the irradiated material, nor the dominant hardening source at various irradiation conditions. Further, there have been relatively few studies that provide quantitative correlation between the TEM-observed radiation-damaged microstructure and the measured hardness change of neutron-irradiated tungsten. In this study, we report on a systematic analysis based on implementing the available microstructural observations of neutron-irradiated tungsten within a dispersed barrier hardening model to address these issues, thereby elucidating the microstructure-property correlation of neutron-irradiated tungsten for application as fusion plasma facing material. 


\section{Materials and Neutron Irradiation}

High purity single crystalline tungsten $(99.999 \%)$ purchased from Goodfellow, USA, was investigated in the present study. The major impurities in the non-irradiated material are 10 ppm O, <10 ppm N, 20 ppm C, <5 ppm S, 3 ppm H, and 140 ppm Cu [23]. SS-J3 tensile test specimens [24] were machined directly from the procured tungsten rod and then exposed to neutron irradiation in the flux trap facility in the High Flux Isotope Reactor (HFIR) at ORNL, USA. The irradiation conditions are summarized in Table 2. The neutron irradiation temperature ranges from 90 to $850^{\circ} \mathrm{C}$ and the radiation doses range from 0.03 to $2.2 \mathrm{dpa}$. In this study, we examined irradiation hardening of tungsten specifically using the Vickers microhardness testing because the tensile data cannot be used to analyze hardening due to the severe degradation in fracture toughness, preventing determination of yield strength [25]. Vickers microhardness testing is an efficient means to assess the mechanical properties of irradiated materials because of the small sampling volume requirement. This technique is especially appropriate for samples with high radioactivity, like the neutron-irradiated tungsten in the present study. Following neutron irradiation, Vickers microhardness testing was performed on one tab of the tensile specimens at room temperature, with an indentation load of $1.96 \mathrm{~N} \mathrm{(200} \mathrm{gf)} \mathrm{and} \mathrm{a} \mathrm{dwell}$ time of $15 \mathrm{~s}$. Microstructure characterization of the irradiated samples were conducted in the low activation materials development and analysis lab (LAMDA) at ORNL by using a JEOM JEM 2100F TEM. TEM foils were prepared using a focused ion beam system (FEI Quanta 3D Dual Beam) operated at 30kV for initial milling and at $5 \mathrm{kV}$ for final thinning. In addition, the Vickers microhardness and microstructural information of the tungsten samples irradiated in Joyo and JMTR [16] [18] [9] [21] [10] were also evaluated in this study for comparison of the impact of the neutron energy spectrum.

\section{Irradiation hardening}

\subsection{Dispersed barrier hardening model}

As noted in Ref. [26], two hardening models have historically been used to describe radiation hardening in metals. The dispersed barrier model is based on straightforward geometrical considerations for obstacles intersecting the dislocation glide plane. It is most appropriate for strong obstacles. An alternative hardening model is developed by FriedelKroupa-Hirsh (FKH) [27] for weak obstacles, where the effective inter-obstacle spacing is increased compared to the dispersed hardening model due to less extensive dislocation bowing prior to obstacle breakaway. The functional dependence of yield strength increase, $\Delta \sigma_{\mathrm{y}}$, on defect cluster size and density for these two limiting cases is given by the following equations, respectively:

$$
\begin{aligned}
& \Delta \sigma_{y}=M \alpha \mu b(N d)^{1 / 2} \\
& \Delta \sigma_{y}=1 / 8 M \mu b d N^{2 / 3}
\end{aligned}
$$

where $\mu$ is the shear modulus of the matrix, which is $161 \mathrm{GPa}$ for tungsten, $\mathrm{b}$ is the magnitude of the dislocation Burgers vector, $\mathrm{M}$ is the Taylor factor, $\mathrm{N}$ is the defect 
number density and $\mathrm{d}$ is the defect diameter, and $\alpha$ is the defect cluster barrier strength. The Schmid factor is used to correlate the critical shear stress resolved on the slip plane to the yield strength in a tensile test if only one specific slip plane is activated in a single crystal. However, the indenter in a hardness test produces a much more complicated and non-uniaxial stress state than a tensile test, which activates multiple slip planes. Therefore, the use of the Taylor factor, which captures the dependence of various slip planes, is more appropriate to convert the critical shear stress into yield stress. The value of 3.06 is recommended for non-textured BCC and FCC polycrystalline metals by Stoller and Zinkle [28]. This value was obtained by averaging the Taylor factors of all possible tensile orientations [29] in a BCC or FCC crystal.

Based on the TEM observations, which will be presented in Section 3.2 and 3.3, dislocation loops, voids, and second phase precipitates are the most commonly observed radiation damaged defect microstructure in neutron-irradiated tungsten. Voids and precipitates are considered as strong obstacles while dislocation loops are usually considered as weak obstacles, although the barrier strength factors proposed for dislocation loops cover a large range $(0.15$ to 0.75$)$ [30]. In addition, previous hardening studies of neutron-irradiated tungsten employed the dispersed hardening model [21] [16]. The use of the same model will enable a direct comparison of the barrier strength factors of various defects between the present and previous studies. Therefore, the dispersed barrier hardening model will be used to assess the measured hardness in the present study, based on the experimentally determined microstructures. The applicability of the FKH model for the weak obstacles (i.e., dislocation loops) will be discussed in more details in Section 4.

In the dispersed barrier hardening model, expressed in Eq. (1), the square-root factor represents the reciprocal of the average distance between obstacles. The $\alpha$ factor accounts for the fact that some obstacles may be partially cut or sheared by the mobile dislocation as it bows out during the glide process. The value of $\alpha$ varies between zero and one, and is strongly dependent on the defect type, size, and temperature [31] [32]. In the present work, the irradiation hardening of tungsten is exclusively ascribed to the existence of irradiation induced dislocation loops, voids, and precipitates, for which the $\alpha$ values need to be identified. $\bar{b}=\frac{a}{2}<111>$ is used in this study with the magnitude of $\mathrm{b}$ set to $0.2741 \mathrm{~nm}$, considering the BCC crystalline structure of tungsten.

Since Eqs. (1) and (2) are provided for yield strength changes, and we have measured the Vickers microhardness, we must also incorporate an appropriate correlation between hardness and yield strength. Tabor [33] derived a linear correlation between the Vickers microhardness and yield strength by examining the stress loaded on the indenter tip in two dimensions (along and perpendicular to the axis of the indenter tip) during the plastic deformation process, expressed in the form of

$$
H_{V}=k \sigma_{y}
$$

with $\mathrm{Hv}$ and $\sigma_{y}$ in $\mathrm{MPa}$, where $\mathrm{k}$ is a constant and is determined as 2.74 in Tabor's theory. Busby [34] summarized the available experimental data of both irradiated austenitic and 
ferritic stainless steels in the open literature and concluded that the values of $\mathrm{k}$ of 3.23 and 3.20, respectively, are most appropriate for austenitic and ferritic stainless steels. Considering that the theoretical analysis was based on an over-simplified model, the linear coefficient obtained from experimental data is used in this study. Therefore, the expression of $\Delta H_{V}=3.20 \sigma_{y}$ will be used due to the BCC lattice structure of both tungsten and the ferritic stainless steels. The final expression of predicted hardening contribution based on the measured defect density, size and type is

$$
\Delta H_{V}=3.20 M \alpha \mu b(N d)^{1 / 2}
$$

Finally, an appropriate superposition law is needed to account for obstacles of different types, size, and number density contained within the irradiated materials. Normally, the root-sum-square superposition law works well when obstacles have similar strengths, and the linear superposition law is better as the strengths become dissimilar [35]. Considering there is no clear physical motivation to support these two superposition laws, the applicability of both laws will be evaluated in the present study.

\subsection{Contribution of intrinsic defects to the irradiation hardening}

When tungsten is subject to neutron irradiation in HFIR, its large thermal neutron capture cross section leads to the generation of Re. Much of the Re produced will, in turn, transmute to Os through subsequent nuclear transmutation reactions. With increasing radiation dose and corresponding increased $\mathrm{Re}$ and Os concentrations, the formation of intermetallic second phase precipitates rich in these elements is observed after the solubility limits are reached. Various TEM studies [18, 36, 37] of tungsten and its alloys irradiated in mixed neutron spectra have shown a large quantity of needle-shaped precipitates when the irradiation dose is greater than $1 \mathrm{dpa}$. The formation of precipitates in neutron-irradiated tungsten complicates the hardening analysis. Prior to evaluating the hardening contribution from precipitates, the barrier strength of the intrinsic defects induced by irradiation, i.e., dislocation loops and vacancy clusters/voids, need to be clarified first.

In Ref. [23], we presented results from an isochronal annealing study of single crystalline tungsten exposed to low dose and low temperature neutron irradiation in HFIR. This particular sample, designated as $1 \mathrm{~W} 25$, was irradiated to $0.03 \mathrm{dpa}$ at $90^{\circ} \mathrm{C}$. The production of transmutation elements during this short irradiation duration is negligible. The coincidence Doppler broadening measurements of the irradiated sample following various annealing stages confirmed this hypothesis. Positron annihilation lifetime spectroscopy (PALS) was utilized to assess the vacancy cluster population in the sample and TEM observations were performed at selected annealing conditions to provide information on the populations of dislocation loops and visible voids. In addition, Vickers microhardness was performed following each annealing condition, thus providing an opportunity to make the link between the microstructure and the mechanical property changes, and enabling us to focus on the hardening contribution from the intrinsic defects. Figure 1 shows the measured Vickers microhardness, as well as the number density of 
large vacancy clusters obtained by PALS and the TEM observed dislocation loop density as a function of the isochronal annealing temperature. The PALS analysis quantified three different types of vacancy clusters, namely, small $(\sim 0.31 \mathrm{~nm})$, intermediate $(\sim$ $0.67 \mathrm{~nm})$, and large $(>0.8 \mathrm{~nm})$ vacancy clusters. As the barrier strength factor is sizedependent, we only consider the hardening contributions from the large vacancy clusters, assuming small and intermediate vacancy clusters have negligible effects on the hardening. TEM observations indicated that dislocation loops were only observed when annealing temperatures were lower than $1000^{\circ} \mathrm{C}$, while small visible voids with a diameter of $\sim 1 \mathrm{~nm}$ appeared when annealing temperatures were above $1000^{\circ} \mathrm{C}$. The hardening was observed to increase following the anneal for temperatures lower than $1000^{\circ} \mathrm{C}$, which we attributed to coarsening of the large, but not yet TEM visible vacancy clusters. The decreasing measured hardness in the temperature regime from 1000 to $1300^{\circ} \mathrm{C}$ was assumed to result from the number density decrease of the large vacancy clusters and voids.

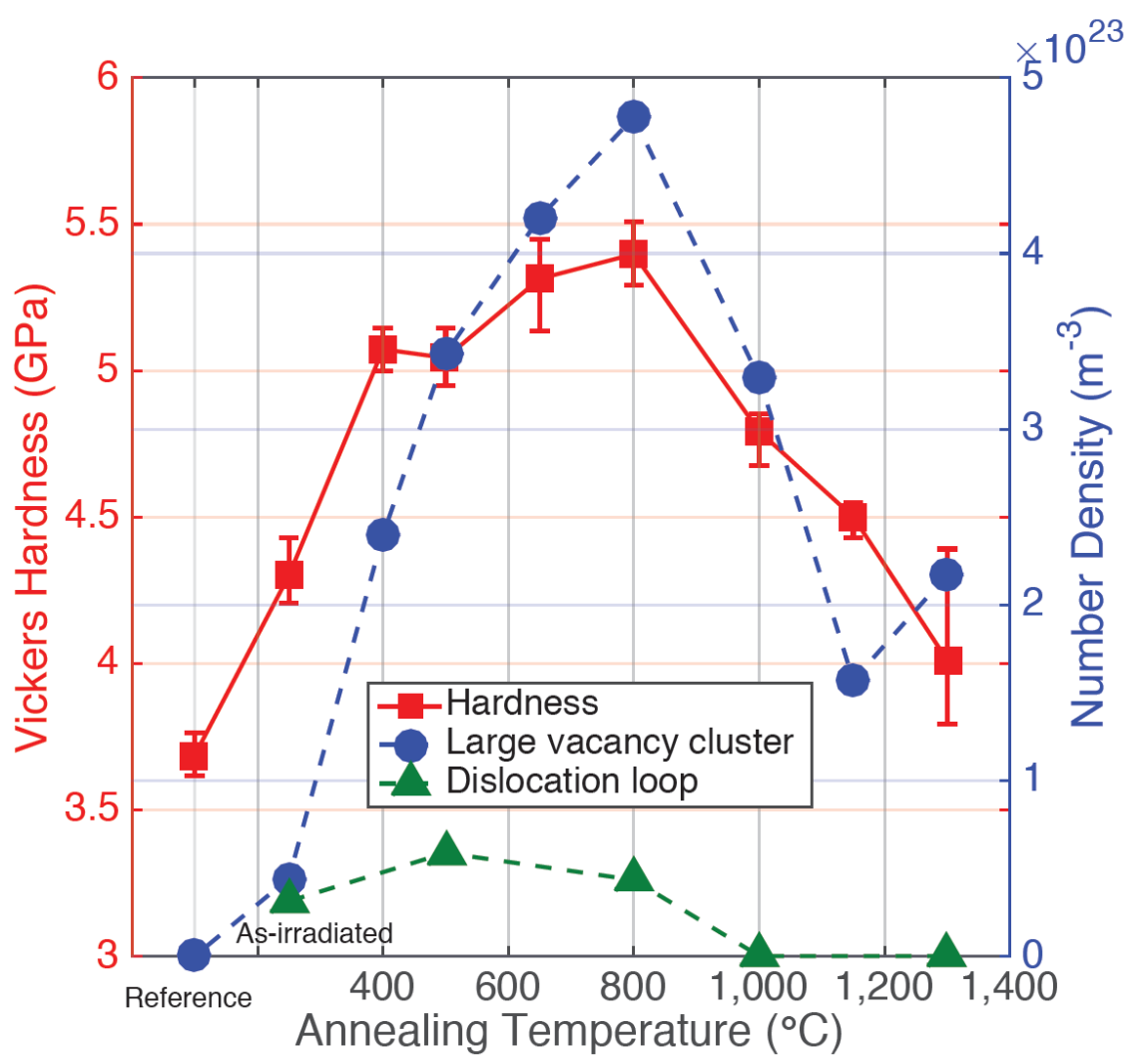

Figure 1. Measured Vickers micorhardness (Red), number density of large vacancy cluster determined by PALS (Blue), and dislocation loop observed by using TEM (Green) at various annealing conditions.

Table 1 lists the experimentally determined number density and size of the dislocation loops, large vacancy clusters, and voids for each annealing condition, in addition to the measured Vickers hardness. In order to apply the dispersed barrier hardening model to quantitatively assess the hardening contributions, we must specify an obstacle strength factor, $\alpha$, of each defect cluster type. Considering the vacancy type defects as a reasonably strong obstacle to dislocation motion [35], the strength factor of large vacancy 
clusters is assigned to be 0.2 , which also is used for the visible voids in TEM observations, given their similar size. Dislocation loops are considered as a slightly weaker obstacle relative to the small voids, therefore, the strength factor of dislocation loops is assumed to be 0.15 in the present work. The input of the available microstructural information listed in Table 1 to Eq. (1) provides a prediction of the hardness. The determination of these $\alpha$ values was also the result of a comparison to ensure good agreement between the modeling prediction and the experimentally measured hardness. The last three columns in Table 1 show a comparison of the measured and predicted hardness data in the annealing study. The use of the root-sumsquare superposition law yields a relatively good agreement with the experimental data, as compared to a linear superposition law. Therefore, this provides confidence in our decision to use the dispersed barrier hardening model throughout the remainder of this analysis, including for the more complicated microstructures that are produced in higher dose irradiation. 
Table 1. Number density and size of irradiation-induced defect clusters in $1 \mathrm{~W} 25\left(90^{\circ} \mathrm{C}, 0.03 \mathrm{dpa}\right)$ following various annealing conditions together with the comparison of measured Vickers hardness and model-predicted hardness

\begin{tabular}{|c|c|c|c|c|c|c|c|c|c|}
\hline \multirow[b]{2}{*}{$\begin{array}{l}\text { Sample } \\
\text { condition }\end{array}$} & \multicolumn{2}{|c|}{ Dislocation loop } & \multicolumn{2}{|c|}{ Large vacancy cluster } & \multicolumn{2}{|c|}{ Void } & \multirow[b]{2}{*}{$\begin{array}{c}\text { Measured } \\
\text { Hardness } \\
\text { Increase } \\
(\mathrm{GPa})\end{array}$} & \multirow[b]{2}{*}{$\begin{array}{l}\text { Modeling } \\
\text { results - } \\
\text { Root-sum- } \\
\text { square } \\
\text { (GPa) }\end{array}$} & \multirow[b]{2}{*}{$\begin{array}{c}\text { Modeling } \\
\text { results - } \\
\text { linear } \\
(\mathrm{GPa})\end{array}$} \\
\hline & $\begin{array}{l}\text { Diameter } \\
(\mathrm{nm})\end{array}$ & $\begin{array}{c}\text { Number } \\
\text { density } \\
\left(\times 10^{22 /}\right. \\
\left.\mathrm{m}^{3}\right) \\
\end{array}$ & $\begin{array}{l}\text { Diameter } \\
(\mathrm{nm})\end{array}$ & $\begin{array}{c}\text { Number } \\
\text { density } \\
\left(\times 10^{22} /\right. \\
\left.\mathrm{m}^{3}\right)\end{array}$ & $\begin{array}{l}\text { Diameter } \\
(\mathrm{nm})\end{array}$ & $\begin{array}{c}\text { Number } \\
\text { density } \\
\left(\times 10^{22} /\right. \\
\left.\mathrm{m}^{3}\right)\end{array}$ & & & \\
\hline Reference & - & - & - & - & - & - & $(3.69)^{*}$ & - & - \\
\hline As-irradiated & 3.08 & 3.12 & & 4.4 & - & - & 0.61 & 0.65 & 0.89 \\
\hline $500^{\circ} \mathrm{C}, 1$ hour & 4.54 & 5.87 & & 34.3 & - & - & 1.35 & 1.33 & 1.88 \\
\hline $800^{\circ} \mathrm{C}, 1$ hour & 5.10 & 4.33 & 0.8 & 47.7 & - & - & 1.71 & 1.41 & 1.97 \\
\hline $1000^{\circ} \mathrm{C}, 1$ hour & - & - & & 11.9 & 10 & 21 & 1.10 & 1.26 & 1.69 \\
\hline $1300^{\circ} \mathrm{C}, 1$ hour & - & - & & 7.7 & 1.0 & 14 & 0.32 & 1.03 & 1.38 \\
\hline
\end{tabular}

*Measured Vickers hardness of reference tungsten sample 


\subsection{Irradiation hardening of tungsten exposed to various neutron energy spectra}

Fukuda et al. [38] summarized the irradiation hardening behavior of tungsten exposed to various neutron energy spectra, including the dependence on neutron fluence and irradiation temperature. The irradiation hardening was indicated by the difference in measured Vickers hardness before and after irradiation. As shown in Figure 2, the irradiation hardening behavior of tungsten is quite dependent on irradiation dose but has very limited temperature dependence, at least for doses less than $5 \mathrm{dpa}$. For the samples irradiated in HFIR, a surprising and very significant hardness increase is observed for radiation dose higher than $0.6 \mathrm{dpa}$. The hardening behavior is very similar for the samples irradiated in JMTR, likely due to the similar neutron energy spectrum of JMTR to HFIR. Additionally, there is almost no difference in the irradiation hardening behavior below 1dpa when comparing the samples irradiated in the fast reactor, Joyo, to the results of the samples subject to a mixed neutron energy spectrum (HFIR and JMTR). However, for the tungsten samples irradiated in the fast neutron spectrum of Joyo there is a suggestion of saturation in the hardness change for dose levels beyond $1 \mathrm{dpa}$. More details on the JMTR and JOYO irradiation results are in Ref. [38].

The number density and size of TEM observed microstructural features, i.e., dislocation loops, voids, and precipitates, in the tungsten samples following neutron irradiation in HFIR, Joyo, or JMTR are summarized in Table 2. Selected TEM images are shown in Figure 3 to demonstrate the commonly observed defect microstructures in tungsten irradiated in HFIR. Dislocation loops were observed in the samples irradiated in the low temperature regime $\left(<500^{\circ} \mathrm{C}\right)$ at dose levels from 0.03 to $1.62 \mathrm{dpa}$. In the temperature range from 500 to $800^{\circ} \mathrm{C}$, the number densities of dislocation loops are at very low levels, or even negligible, for tungsten irradiated to a dose between 0.15 and $2.2 \mathrm{dpa}$. This observation is consistent with the high mobility of dislocation loops [39] [40] at higher temperatures that promotes recombination with vacancy clusters or annihilation at other sinks, given that the migration energy of interstitial dislocation loops are very small, $0.013 \mathrm{eV}[15]$. Therefore, the temperature dependence of the dislocation loops is more significant in comparison to the dose dependence for the tungsten samples exposed to neutron irradiation in HFIR. Similar behavior can also be observed for the samples irradiated in Joyo.

In addition, voids were observed in tungsten samples irradiated in the temperature range from 724 to $800^{\circ} \mathrm{C}$, covering the dose levels of 0.15 to $2.2 \mathrm{dpa}$. Since the vacancy migration energy is quite large in tungsten, with reported values ranging from 1.34 to $2.44 \mathrm{eV}[15,41][42]$, the relatively low vacancy diffusivity will require higher temperatures to induce the interaction of vacancy clusters to promote void nucleation and formation. In addition to the voids and dislocation loops, large $\mathrm{W}$-Re-Os precipitates were also observed in samples irradiated to higher dose levels (>1 dpa) in HFIR, presumably due to the large thermal neutron flux. The temperature dependence of the precipitate distributions was observed to be insignificant in comparison with its dose dependence. It is noted that the TEM-visible precipitates in HFIR samples are needleshaped and the sizes shown in Table 2 are the measured lengths of these precipitates. The 
crystal structures and coherency to the matrix are currently being examined and the results will be published elsewhere soon. For simplification, the length is assumed to be the characteristic size of the precipitates used in the dispersed barrier hardening model. For the samples irradiated in JMTR at 600 and $800^{\circ} \mathrm{C}$ to $0.15 \mathrm{dpa}$, both voids and dislocation loops were observed. No precipitation was visible in these two samples irradiated in JMTR with a mixed neutron energy spectrum, likely due to the relatively small neutron exposure. When tungsten samples were irradiated in the fast reactor, Joyo, the observed irradiation defect microstructures consisted only of dislocation loops and voids, except for the case of $750^{\circ} \mathrm{C}$ irradiation. It is noted that the number density of loops in these Joyo samples were low for irradiation temperatures from 580 to $760^{\circ} \mathrm{C}$, consistent with the observations of the HFIR samples irradiated at high temperatures, i.e., greater than $700^{\circ} \mathrm{C}$.

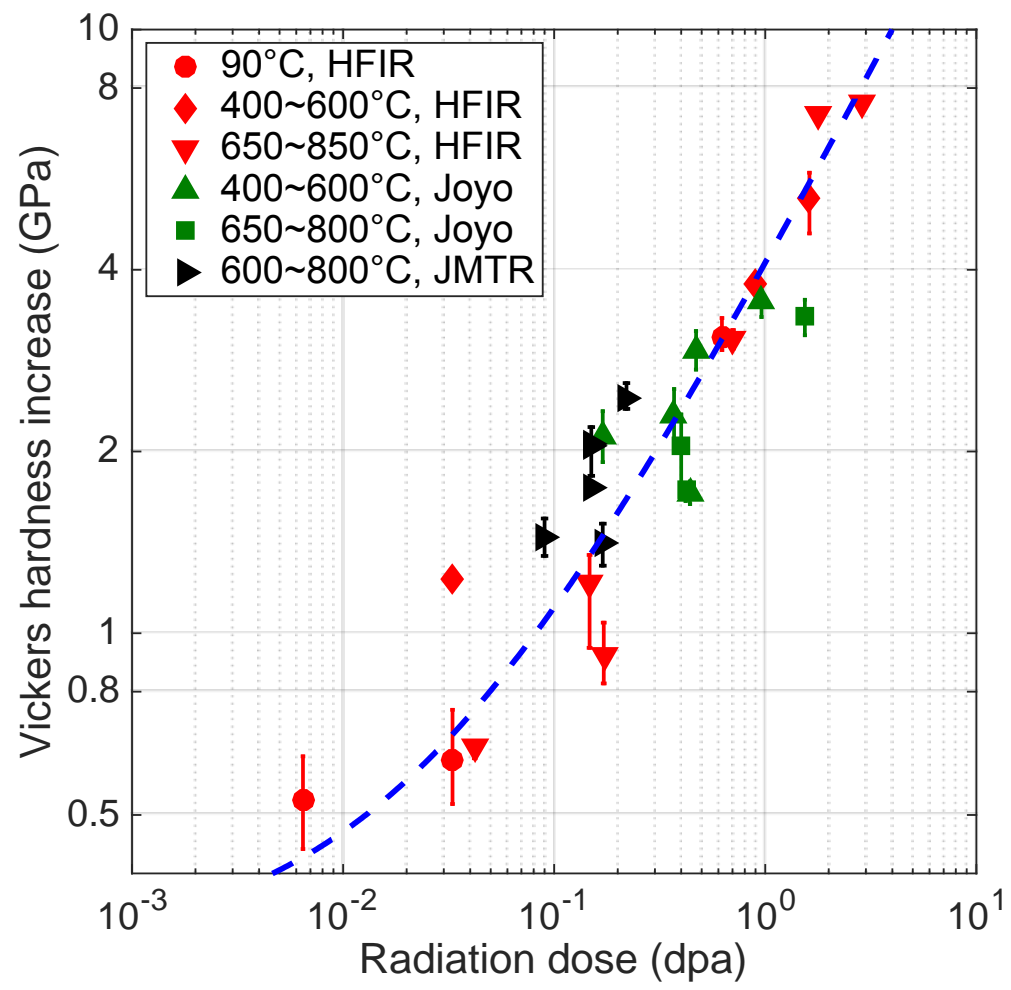

Figure 2 Dose and temperature dependence of irradiation hardening of pure tungsten irradiated in the HFIR, Joyo, and JMTR. The dashed line is plotted to guide the eye.

In order to calculate the hardness with a dispersed barrier hardening model, the obstacle strength factor, $\alpha$, needs to be specified for all the TEM-visible defects contained within the neutron irradiated tungsten samples. In this study, we have assumed that these obstacle strength values are a function of defect type and size. In determining these $\alpha$ values, we have assumed that voids are strong obstacles to dislocation motion in comparison with dislocation loops [35] and the large TEM-observed W-Re-Os precipitates are also strong obstacles [18] [30]. Following the same methodology stated in Section 3.2, the $\alpha$ values of various defects are obtained and listed in Table 3. The calculated hardness values by using the two different superposition laws are shown in the last two columns of Table 2 . 
Figure 4 shows the comparison between experimental data and the modeling predictions by using root-sum-square (Figure 4 (a)) and linear (Figure 4 (b)) superposition laws, respectively. The results indicate that no obvious difference was observed for these two superposition laws in the present study while the root-sum-square law has a slightly better performance.

Based on the empirically determined barrier strength of each defect type using the linear superposition law, the individual contribution of each defect to the hardening in this case can be computed, as shown in Figure 5. Because of the nonlinear relationship of the contributions of each type of defect in the root-sum-square case, individual contributions are not shown here. Several important features of the system can be derived from Figure 5 .

For the tungsten samples irradiated in HFIR, the predicted hardness has a relatively good agreement with the experimental data in the low dose $(<0.1 \mathrm{dpa})$ and high dose regions $(>0.6 \mathrm{dpa}$ ) while significant discrepancies are observed for the dose range from 0.1 to 0.6 dpa. Based on this analysis, coupled to the aforementioned TEM observations, we conclude that the irradiation hardening of neutron-irradiated tungsten in low dose regime derives from the existence of dislocation loops. The dominant hardening feature in the high radiation dose region is the large $\mathrm{W}$-Re-Os precipitates. For those samples irradiated to intermediate dose, only dislocation loops and voids were TEM-visible and thus considered in the hardening model, leading to much smaller hardness predictions than measured values. This implies that a substantial volume fraction of microstructural features are invisible in TEM observations, and correspondingly, are not captured in the hardening model. The most likely cause is the formation of very small transumutationinduced W-Re-Os clusters. A recent APT study on the neutron irradiated tungsten [36] supports this view, as that characterization observed a relatively large number density of W-Re-Os clusters after being exposed to HFIR neutron irradiation to a dose of $0.2 \mathrm{dpa}$ at $750^{\circ} \mathrm{C}$.

Additionally, when applying the hardening model to the tungsten samples irradiated in JMTR, the model also under estimated the measured hardness increase. Considering that JMTR has a mixed neutron spectrum, similar to HFIR, the production of transmutant elements is expected. For the two samples irradiated to 0.15 dpa in JMTR, only dislocation loops and voids were observed in TEM observations. Thus, it is tempting to conclude that, just like the tungsten samples irradiated in HFIR to the intermediate dose range, a hardening contribution from small, TEM invisible precipitates was not captured, resulting in the underestimation of the hardness increase. Moreover, for the samples irradiated in Joyo, which has a fast neutron energy spectrum, the model prediction and the experimental data have a relatively good agreement in comparison with the tungsten samples irradiated in HFIR and JMTR. For tungsten exposed to a fast neutron spectrum, the generation of transmutant elements, and correspondingly, the formation of Re-Os-W precipitates are much less than when irradiated in a mixed neutron energy spectrum. Therefore, the hardening contribution from the $\mathrm{W}$-Re-Os precipitates could be neglected 
while the dislocation loops and voids in irradiated tungsten are the dominant hardening sources.
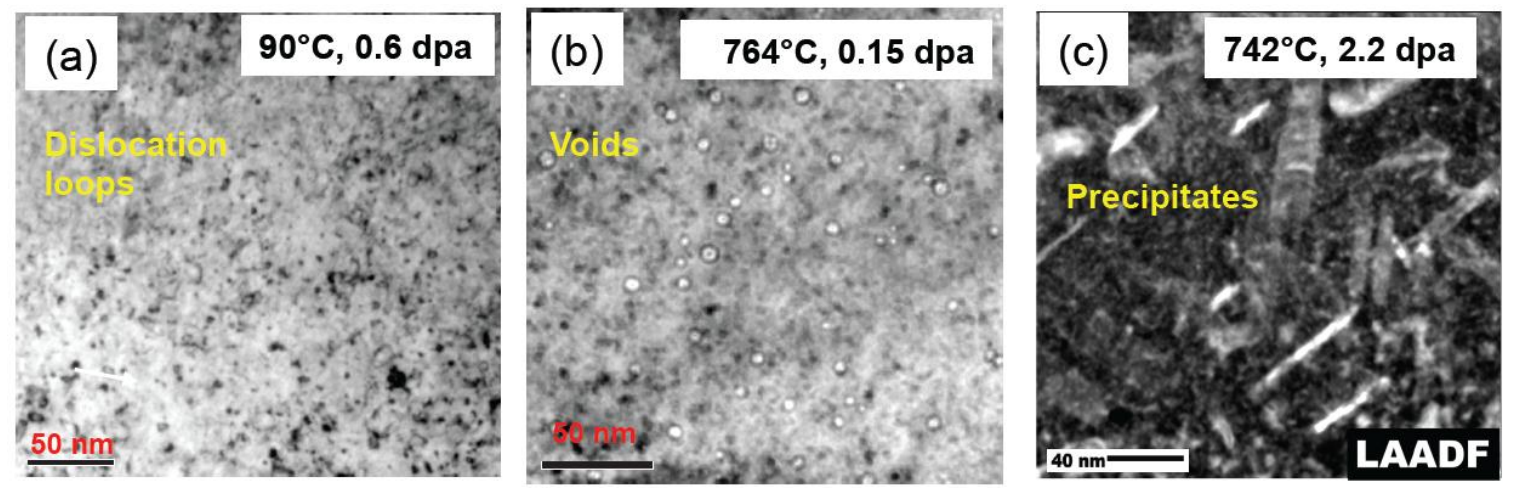

Figure 3. (a) Bright field TEM image of tungsten irradiated at $90^{\circ} \mathrm{C}$ to $0.6 \mathrm{dpa}$; (b) Bright field TEM image of tungsten irradiated at $764^{\circ} \mathrm{C}$ to $0.15 \mathrm{dpa}$; (c) LAADF TEM image of tungsten irradiated at $742^{\circ} \mathrm{C}$ to 2.2 dpa. All tungsten samples are single crystalline with an orientation of (110) and were irradiated in HFIR. 
Table 2 Number density and size of irradiation-induced defects in tungsten subject to neutron exposure in HFIR, Joyo, and JMTR together with the comparison of measured Vickers hardness and model-predicted hardness

\begin{tabular}{|c|c|c|c|c|c|c|c|c|c|c|c|c|}
\hline \multirow[b]{2}{*}{ Reactors } & \multirow[b]{2}{*}{$\begin{array}{l}\text { Irradiation } \\
\text { temperature } \\
\quad\left({ }^{\circ} \mathrm{C}\right)\end{array}$} & \multirow[b]{2}{*}{$\begin{array}{c}\text { Neutron } \\
\text { fluence } \\
\left(10^{25}\right. \\
\mathrm{n} / \mathrm{m}^{2}, \\
\mathrm{E}>0.1 \mathrm{M} \\
\mathrm{eV})\end{array}$} & \multirow[b]{2}{*}{$\begin{array}{l}\text { Radiation } \\
\text { dose } \\
\text { (dpa) }\end{array}$} & \multicolumn{2}{|c|}{ Dislocation loop } & \multicolumn{2}{|c|}{ Void } & \multicolumn{2}{|c|}{ Precipitate } & \multirow[b]{2}{*}{$\begin{array}{c}\text { Measured } \\
\text { hardness } \\
\text { increase } \\
(\mathrm{GPa})\end{array}$} & \multirow[b]{2}{*}{$\begin{array}{l}\text { Predicted } \\
\text { hardness } \\
\text { increase } \\
\text { Root-Sum- } \\
\text { Square } \\
\text { (GPa) }\end{array}$} & \multirow[b]{2}{*}{$\begin{array}{c}\text { Predicted } \\
\text { hardness } \\
\text { increase } \\
\text { Linear } \\
(\mathrm{GPa})\end{array}$} \\
\hline & & & & $\begin{array}{c}\text { Average } \\
\text { size } \\
(\mathrm{nm})\end{array}$ & $\begin{array}{c}\text { Number } \\
\text { density } \\
\left(\times 10^{22}\right. \\
\left.\mathrm{m}^{-3}\right)\end{array}$ & $\begin{array}{l}\text { Average } \\
\text { size }(\mathrm{nm})\end{array}$ & $\begin{array}{c}\text { Number } \\
\text { density } \\
\left(\times 10^{22}\right. \\
\left.\mathrm{m}^{-3}\right)\end{array}$ & $\begin{array}{l}\text { Average } \\
\text { size }(n m)\end{array}$ & $\begin{array}{c}\text { Number } \\
\text { density } \\
\left(\times 10^{22}\right. \\
\left.\mathrm{m}^{-3}\right)\end{array}$ & & & \\
\hline \multirow{8}{*}{ HFIR } & 90 & 2 & 0.6 & 4.98 & 4.55 & - & - & - & - & 3.10 & 0.98 & 0.98 \\
\hline & 397 & 0.1 & 0.03 & 5.12 & 2.7 & - & - & - & - & 1.23 & 0.77 & 0.77 \\
\hline & 467 & 2 & 0.6 & 5.38 & 3.1 & - & - & - & - & 3.80 & 0.83 & 0.83 \\
\hline & 724 & 2 & 0.6 & 6.37 & $<0.01$ & 3.03 & 1.22 & - & - & 3.07 & 0.79 & 0.84 \\
\hline & 742 & 7 & 2.2 & - & - & 5.28 & 0.07 & 20 & 4.8 & 7.56 & 8.06 & 8.38 \\
\hline & 764 & 0.5 & 0.15 & 6.56 & $<0.01$ & 3.49 & 0.5 & - & - & 0.92 & 0.54 & 0.60 \\
\hline & 500 & 5.4 & 1.62 & 2.9 & 3.3 & - & - & 5.7 & 8.6 & 5.24 & 5.78 & 6.39 \\
\hline & 800 & 5.9 & 1.77 & - & - & 3.8 & 0.8 & 16.3 & 3.6 & 7.21 & 6.33 & 7.01 \\
\hline \multirow{2}{*}{$\mathrm{JMTR}^{[43][20]}$} & 600 & 0.81 & 0.15 & 7.9 & 4.6 & 1.3 & 6.4 & - & - & 2.06 & 1.58 & 2.23 \\
\hline & 800 & 0.81 & 0.15 & 8.5 & 1.1 & 1.9 & 4.2 & - & - & 1.74 & 1.15 & 1.59 \\
\hline \multirow{7}{*}{ Joyo ${ }^{[44][21]}$} & 400 & 1.1 & 0.17 & 2.8 & 2.0 & 1.8 & 19.5 & - & - & 2.12 & 2.08 & 2.52 \\
\hline & 531 & 3.4 & 0.44 & 5.4 & 1.1 & 1.3 & 25.3 & - & - & 1.71 & 2.03 & 2.46 \\
\hline & 538 & 7.8 & 0.96 & 4.7 & 4.7 & 2.1 & 49.0 & - & - & 3.56 & 4.28 & 5.13 \\
\hline & 583 & 3.7 & 0.47 & 5.4 & 0.2 & 2.4 & 13.8 & - & - & 2.95 & 2.37 & 2.58 \\
\hline & 740 & 2.7 & 0.4 & 12.2 & 0.3 & 2.9 & 12.7 & - & - & 2.05 & 2.53 & 2.88 \\
\hline & 750 & 12 & 1.54 & - & - & 4.7 & 12.0 & - & - & 3.34 & 4.12 & 4.12 \\
\hline & 756 & 3.3 & 0.42 & 5.6 & 0.1 & 2.5 & 12.1 & - & - & 1.72 & 2.26 & 2.41 \\
\hline
\end{tabular}

Table 3 Barrier strength factor as a function of defect type and size used in the dispersed barrier hardening model

\begin{tabular}{|c|c|c|c|c|c|c|}
\hline \multirow{2}{*}{ Defects } & \multirow{2}{*}{$\begin{array}{c}\text { Dislocation } \\
\text { loops }\end{array}$} & \multicolumn{4}{|c|}{ Voids } & \multirow[b]{2}{*}{ Precipitates } \\
\hline & & $1 \sim 2 \mathrm{~nm}$ & $2 \sim 3 \mathrm{~nm}$ & $3 \sim 4 \mathrm{~nm}$ & $>4 n m$ & \\
\hline $\begin{array}{c}\text { Barrier } \\
\text { strength } \\
\text { factor }(\alpha)\end{array}$ & 0.15 & 0.25 & 0.30 & 0.35 & 0.40 & 0.60 \\
\hline
\end{tabular}


(a)

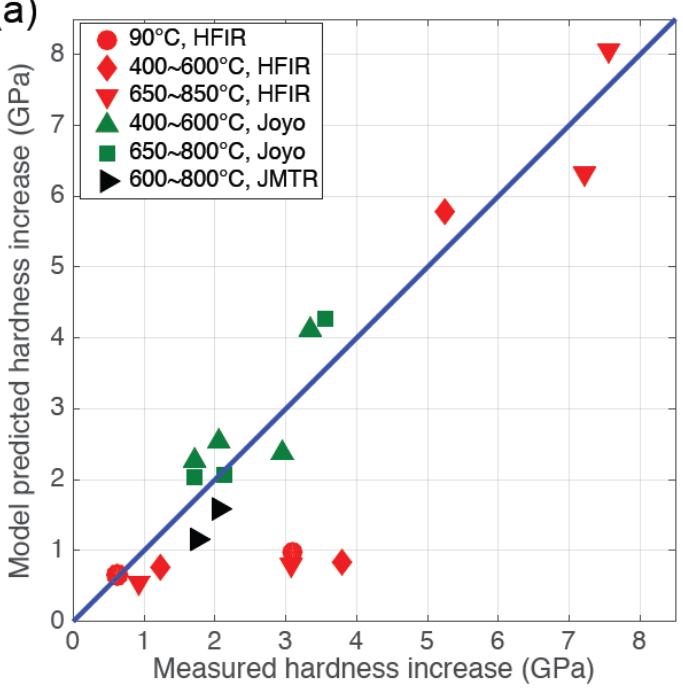

(b)

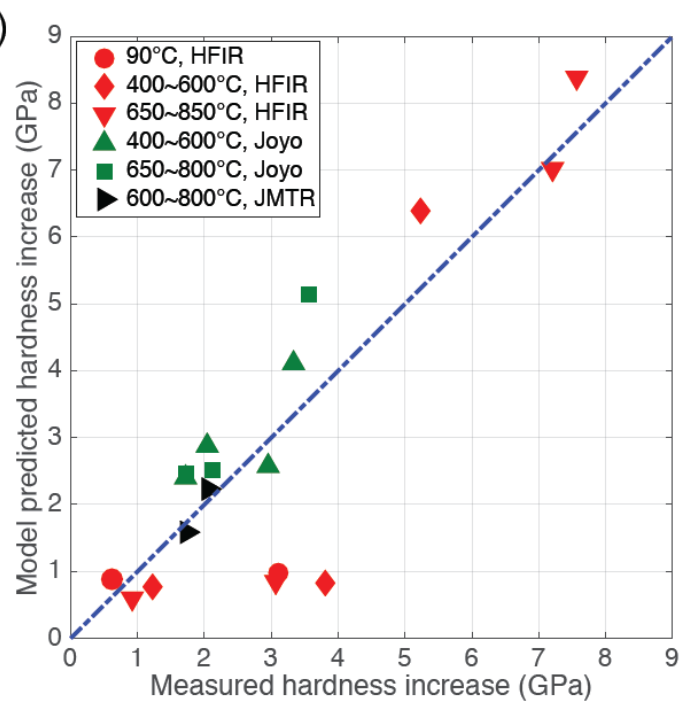

Figure 4 Comparison of experimentally measured Vickers hardness increase and hardening model predictions by using (a) root-sum-square and (b) linear superposition laws, respectively.

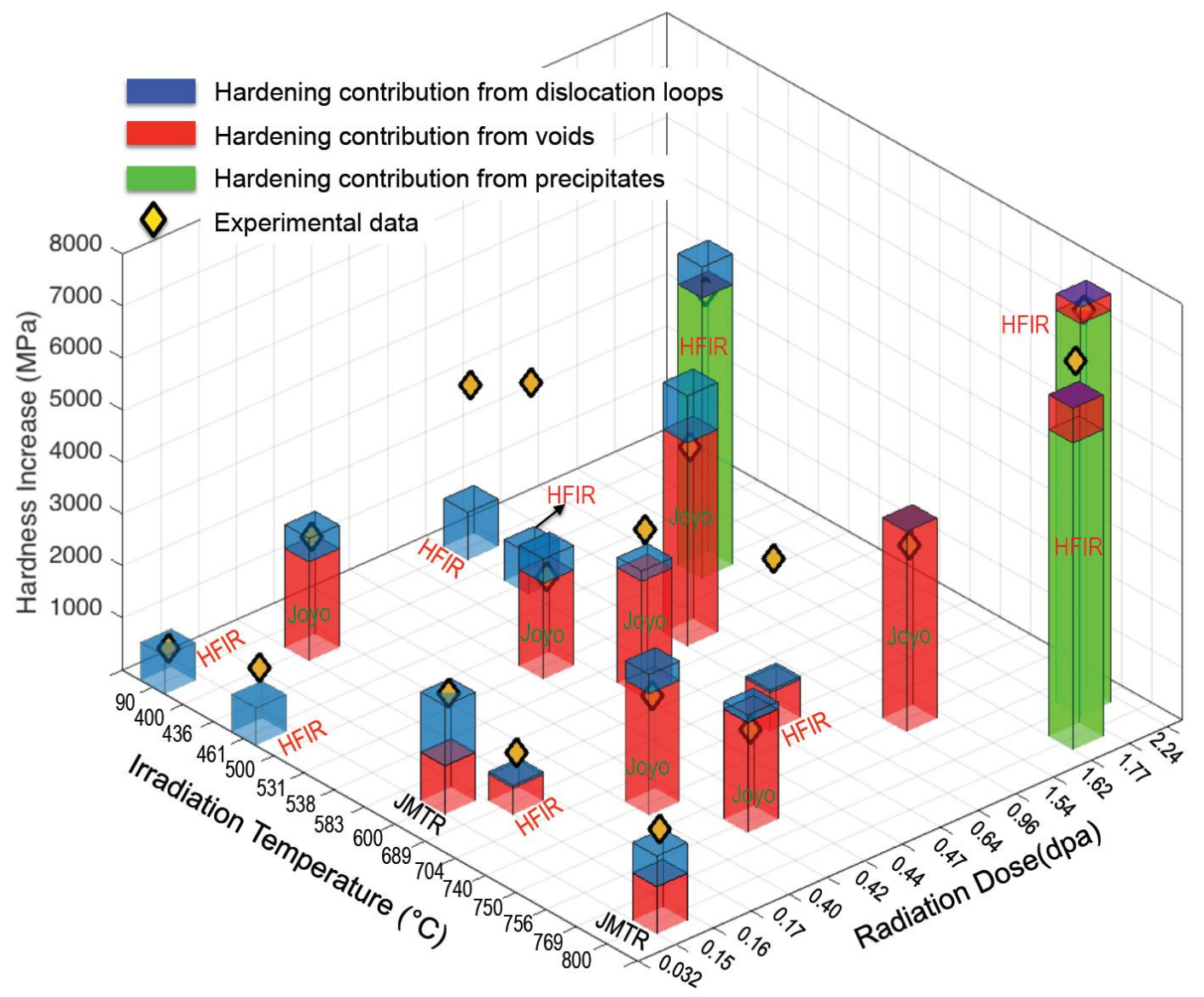


Figure 5 Radiation-induced hardening contributions due to different measured defects based on the linear superposition of the dispersed barrier hardening model for the samples listed in Table 2. The $\mathrm{x}$ - and $\mathrm{y}$-axes are not linear scaled.

\section{Discussion}

As discussed in Section 3.1, the FKH model may be more appropriate for many radiationinduced small defect clusters that are weak obstacles to dislocation motion. According to Zinkle [26], this model is adequate for barrier strengths up to about one quarter of the Orowan (impenetrable obstacle) limit, i.e., $\alpha<0.25$. The dispersed barrier model is more appropriate for barrier strengths of $0.25<\alpha<1.0$. It is commonly accepted that dislocation loops are considered as weak obstacles [30]. Based on the barrier strength factors determined in this study, listed in Table 3, this conclusion can further be confirmed. In order to examine the applicability of the FKH model, a combined model is used to predict the hardness increase of tungsten exposed to neutron irradiation, where the hardening contribution of dislocation loops is computed by using FKH model and the dispersed barrier hardening model is used for other defects' hardening contribution. The individual hardening contribution of different defects were then integrated by using the root-sumsquare superposition law. The comparison of the experimentally measured Vickers micro-hardness and the combined model predictions is shown in Figure 6. It is evident that the application of the FKH model to the cases having dislocation loops as the dominant hardening source (hollow data points in Figure 6) significantly underestimates the hardness increase in comparison with the results obtained from the dispersed barrier hardening model as shown in Figure 4 (a). Therefore, the FKH hardening model is inappropriate in the present study on irradiation hardening of neutron-irradiated tungsten. 


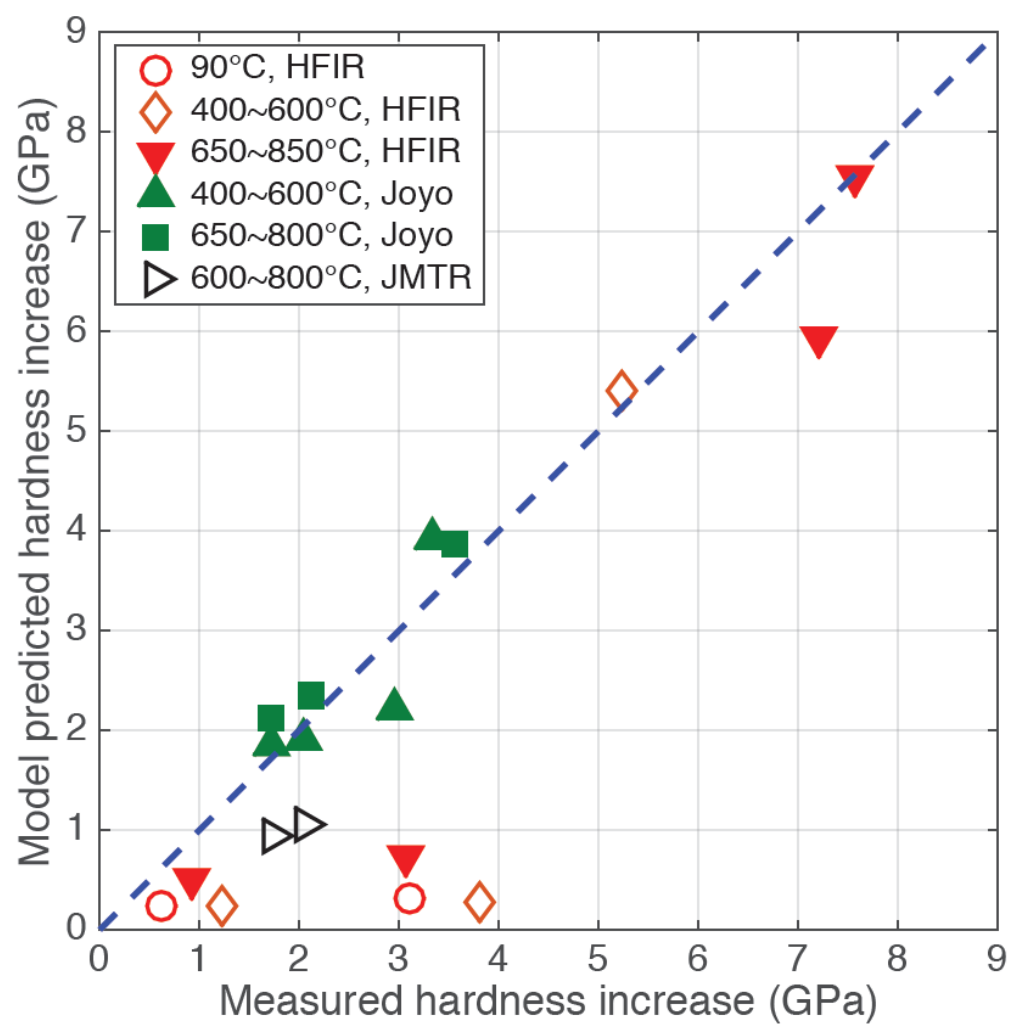

Figure 6. Comparison of experimentally measured Vickers hardness increase and the combined hardening model predictions (dispersed barrier hardening model and FKH model) by using the root-sum-square superposition law. The hollow data points indicate the cases which have significant difference compared to the same conditions shown in Figure 4 (a).

In the present study, a linear relationship between observed microstructure and the measured hardness was obtained based on the dispersed hardening model. The linear expression was also applied in previous studies on irradiation hardening of neutron irradiated tungsten and its alloys [16] [21] except that the coefficient is different, expressed in the form of

$$
\Delta H_{V}=6 \alpha \mu b(N d)^{1 / 2}
$$

with $\mathrm{Hv}$ and $\sigma_{y}$ in MPa. The equation used in these studies was originally described in Ref. [45]. When converting the critical shear stress, which is required to move dislocations through a field of obstacles, to an equivalent uniaxial tensile stress, a coefficient of 2 was used. However, Stoller and Zinkle [28] pointed out that the conversion factor of 2 is not right due to the inappropriate application of the Tresca yield criteria and concluded that the Taylor factor with a value of 3.06 is the correct parameter. Therefore, the barrier strength factors used in previous studies are overestimated by $\sim 1.6$ times the values used in the present study. In Ref. [21], the barrier strength factors of dislocation loop and void are set to be 0.2 and 0.6 , respectively, without considering the size dependence. In order to obtain the same hardness predictions by using the corrected equation, Eq. (4), these values must correspondingly be modified to 0.12 and 0.38 , respectively, which are close to the $\alpha$ values used in the present study for dislocation 
loops $(\alpha=0.15)$ and large voids $(\alpha=0.4)$ with a diameter greater than $4 \mathrm{~nm}$. Typical experimental values of $\alpha$ for the TEM-visible dislocation loops in neutron irradiated BCC metals are covering a large range from 0.15 up to 0.75 [46] [47]. Tan [30] summarized that the strength factors of cavities and precipitates could be in the range of $0.01 \sim 1$ and $0.2 \sim 0.6$, respectively. The barrier strength factors used in the present study, listed in Table 3, fall in these ranges.

It is emphasized here that the irradiation hardening derives from all the defects contained within the materials. However, the hardening model only includes hardening contributions from the TEM-visible defects. Correspondingly, most literature studies assume that any microstructural features that are not visible in the TEM have negligible contributions to the hardening increases of materials following irradiation. This works well for most of the cases in the literature. However, as noted in the previous section, the tungsten specimens irradiated in HFIR and JMTR to an intermediate dose range from about 0.1 to $3 \mathrm{dpa}$, exhibit considerably larger hardness increases than cannot be captured by a dispersed barrier hardening model informed by a microstructure consisting only of the dislocation loops and voids observed in TEM. In this case, we believe that the dominant hardening contribution is from TEM-invisible precipitates, which form due to neutron-induced transmutation of tungsten into rhenium and osmium, and the resistance to dislocation motion of these precipitate clusters. Actually, the generation of solid transmutant element, Re and Os, is a significant issue for neutron-irradiated tungsten. For example, in the tungsten sample irradiated in HFIR, pure tungsten is transformed to WRe or W-Re-Os alloys as the neutron fluence increases. Previous calculations by Greenwood and Garner [48] indicate that pure tungsten is converted to W-8\%Re-15\%Os alloys after $10 \mathrm{dpa}$ in HFIR. Therefore, the material of interest is no longer pure tungsten. In addition to the formation of precipitates, the shear modulus will also change as the material composition changes. Furthermore, even below the solubility limit, the transmutant elements may produce solid solution hardening of tungsten.

For example, Moteff and Rau [13] showed that the increase in the $1100^{\circ} \mathrm{C}$ creep strength of tungsten irradiated at $70^{\circ} \mathrm{C}$ to various radiation dose levels is a function of the $\mathrm{Re}$ content, following a parabolic power law. Fukuda et al. [21] measured the Vickers hardness of W-Re alloys at room temperature and found that the hardness increases as the $\mathrm{Re}$ content increases, and the increase approximately follows a linear dependence on $\mathrm{Re}$ concentration. In order to investigate the solution hardening of BCC Fe matrix, a solid solution strengthening model [49] was developed by establishing a linear correlation between the composition of the solutes in the matrix and the resultant change in yield strength. One particular observation is that interstitial solute species have a strength factor that is 2 to 3 orders of magnitude greater than that of substitutional solutes. Considering the BCC crystalline structure of both $\mathrm{W}$ and $\mathrm{Fe}$, a similar solid solution strengthening behavior is expected for tungsten irradiated in HFIR. In order to get a more accurate prediction of the irradiation hardening, the solution hardening effect should be considered in the future study. More work is also needed to identify the population of the small precipitates in the tungsten samples irradiated in HFIR to intermediate doses and such information will be included in the hardening model to overcome the current gap between modeling and experimental data. 


\section{Conclusion}

Irradiation hardening of tungsten exposed to neutron irradiation with differing neutron energy spectra was investigated through analysis of the hardness changes using a dispersed barrier hardening model informed by the experimentally-observed microstructures in irradiated tungsten using TEM. An annealing study of single crystalline tungsten following low dose and low temperature neutron irradiation was used to identify the hardening contribution of vacancy clusters and dislocation loops. The results indicated that the barrier strength factors of the large vacancy clusters and TEMvisible dislocation loops, the two major hardening sources during the low-dose irradiated sample subject to isochronal annealing, were determined as 0.2 and 0.15 , respectively. Vickers microhardness testing at room temperature of tungsten following various irradiation conditions showed continuously increasing hardness with increasing radiation dose for tungsten irradiated in HFIR and JMTR, while the hardness increase of tungsten irradiated in Joyo appears to saturate for radiation doses above $1 \mathrm{dpa}$. This analysis reveals the dominant hardening source at various irradiation conditions. For the samples irradiated in HFIR, dislocation loops and voids are the major contributor to the irradiation hardening at low dose levels, while intermetallic second phase precipitation dominate for doses greater than $0.6 \mathrm{dpa}$, leading to a tremendous hardness increase. The model fails to predict accurate hardness increase in the intermediate dose range, which we interpret result from TEM-invisible precipitates or even super-saturated solid solutions of transmutant rhenium or osmium. Future work will be dedicated to investigating the transmutant element clusters in this radiation dose range to verify the hypothesis. For the samples irradiated in Joyo and JMTR (those having detailed microstructure information), the major hardening contributors are TEM-visible dislocation loops and voids. The barrier strength factors were also determined for the observed radiation defects, which could be applied to predicting the irradiation hardening of tungsten. The strength factor of dislocation loop is determined as 0.15 while the $\alpha$ values for voids are a function of void size, with a range from 0.25 to 0.4 . The $\mathrm{W}$-Re-Os precipitates are strong obstacles to dislocation motion with $\alpha$ value of 0.6. Overall, this work is expected to provide insights into the underlying mechanisms controlling the embrittlement of tungsten exposed to neutron irradiation and will benefit the $R \& D$ of plasma facing materials in fusion reactors.

\section{Acknowledgement}

The work presented in this paper was partially supported by Laboratory Directed R\&D funds at ORNL. The research was also sponsored by the US Department of Energy Office of Fusion Energy Science under grants DE-AC05-00OR22725 with UT-Battelle LLC and grant DOE-DE-SC0006661 at the University of Tennessee, Knoxville, and by the US-Japan PHENIX project under contract NFE-13-04478, with UT-Battelle LLC.

\section{References}


1. Rieth, M., et al., Recent progress in research on tungsten materials for nuclear fusion applications in Europe. Journal of Nuclear Materials, 2013. 432(1-3): p. 482-500.

2. Ueda, Y., K. Tobita, and Y. Katoh, PSI issues at plasma facing surfaces of blackets in fusion reactors. Journal of Nuclear Materials, 2003. 313-316: p. 32-41.

3. Zinkle, S.J., Fusion materials science: Overview of challenges and recent progress. Physics of Plasmas, 2005. 12(5): 058101.

4. Wirth, B.D., et al., Fusion materials modeling: Challenges and opportunities. MRS Bulletin, 2011. 36(03): p. 216-222.

5. Bloom, E.E., S.J. Zinkle, and F.W. Wiffen, Materials to deliver the promise of fusion power - progress and challenges. Journal of Nuclear Materials, 2004. 329333: p. 12-19.

6. Wurster, S., et al., Recent progress in $R \& D$ on tungsten alloys for divertor structural and plasma facing materials. Journal of Nuclear Materials, 2013. 442(1-3): p. S181-S189.

7. Greenwood, R.L. and K.R. Smither, SPECTER: Neutron damage calculations for materials irradiations. 1985. ANL/FPP/TM-197.

8. Fujitsuka, M., et al., Effect of neutron irradiation on thermal diffusivity of tungsten-rhenium alloys. Journal of Nuclear Materials, 2000. 283-287: p. 11481151.

9. Hasegawa, A., et al., Property change mechanism in tungsten under neutron irradiation in various reactors. Journal of Nuclear Materials, 2011. 417(1-3): p. 491-494.

10. Fukuda, M., et al., Property change of advanced tungsten alloys due to neutron irradiation. Journal of Nuclear Materials, 2013. 442(1-3): p. S273-S276.

11. Davis, J.W. and P.D. Smith, ITER Material properties handbook. Journal of Nuclear Materials, 1996. 233-237: p. 1593-1596.

12. Sikka, V.K. and J. Moteff, Identification of alpha-Mn crystal structure in neutron irradaited W-Re alloy. mEtallurgical Transactions, 1974. 5: p. 1514-1517.

13. Rau, R.C. and J. Moteff, Annealing of defect clusters in irradiated tungsten. Radiation Effects, 1971. 8(1-2): p. 99-105.

14. Williams, R.K., et al., Irradiation induced precipitation in tungsten based, W-Re alloys. Metallurgical Transactions A, 1983. 14A: p. 655-666.

15. Becquart, C.S., et al., Microstructural evolution of irradiated tungsten: Ab initio parameterisation of an OKMC model. Journal of Nuclear Materials, 2010. 403(13): p. 75-88.

16. Tanno, T., et al., Microstructure Development in Neutron Irradiated Tungsten Alloys. Materials Transactions, 2011. 52(7): p. 1447-1451.

17. Ferroni, F., et al., High temperature annealing of ion irradiated tungsten. Acta Materialia, 2015. 90: p. 380-393.

18. Hasegawa, A., et al., Neutron irradiation effects on tungsten materials. Fusion Engineering and Design, 2014. 89(7-8): p. 1568-1572.

19. $\quad$ Snead, L.L., To be published. 2016.

20. Hasegawa, A., et al., Neutron Irradiation Behavior of Tungsten. Materials Transactions, 2013. 54(4): p. 466-471. 
21. Fukuda, M., et al., Effects of Re Content and Fabrication Process on Microstructural Changes and Hardening in Neutron Irradiated Tungsten. Materials Transactions, 2012. 53(12): p. 2145-2150.

22. Fukuda, M., et al., Microstructural development of tungsten and tungstenrhenium alloys due to neutron irradiation in HFIR. Journal of Nuclear Materials, 2014. 455(1-3): p. 460-463.

23. $\mathrm{Hu}, \mathrm{X}$., et al., Defect evolution in single crystalline tungsten following low temperature and low dose neutron irradiation. Journal of Nuclear Materials, 2016. 470: p. 278-289.

24. Gussev, M.N., et al., Role of Scale Factor During Tensile Testing of Small Specimens. 2014: p. 1-19.

25. Garrison, L.M., et al., Irradiation Effects in Tungsten-Copper Laminate Composite. 2015. doe/er-0313/59.

26. Zinkle, S.J. and Y. Matsukawa, Observation and analysis of defect cluster production and interactions with dislocations. Journal of Nuclear Materials, 2004. 329-333: p. 88-96.

27. Kroupa, F. and P.B. Hirsch, Elastic interaction between prismatic dislocation loops and straight dislocations. Discussions of the Faraday Society, 1964. 38: p. 49-55.

28. Stoller, R.E. and S. Zinkle, On the relationship between uniaxial yield strength and resolved shear stress in polycrystalline materials. Journal of Nuclear Materials, 2000. 283-287: p. 349-352.

29. Kocks, U.F., The relation between polycrystal deformation and single-crystal deformation. Metallurgical Transactions, 1970. 1: p. 1121-1143.

30. Tan, L. and J.T. Busby, Formulating the strength factor $\alpha$ for improved predictability of radiation hardening. Journal of Nuclear Materials, 2015. 465: p. 724-730.

31. Field, K.G., et al., Radiation tolerance of neutron-irradiated model $\mathrm{Fe}-\mathrm{Cr}-\mathrm{Al}$ alloys. Journal of Nuclear Materials, 2015. 465: p. 746-755.

32. $\mathrm{Hu}, \mathrm{X}$., et al., Modeling of irradiation hardening of iron after low-dose and lowtemperature neutron irradiation. Modelling and Simulation in Materials Science and Engineering, 2014. 22(6): 065002.

33. Tabor, D., The physical meaning of indentation and scratch hardness. British Journal of Applied Physics 1956. 7: p. 159-166.

34. Busby, J.T., M.C. Hash, and G.S. Was, The relationship between hardness and yield stress in irradiated austenitic and ferritic steels. Journal of Nuclear Materials, 2005. 336(2-3): p. 267-278.

35. Was, G.S., Fundamentals of radiation materials science. 2007, New York: Springer.

36. Edmondson, P.D., To be published, 2016.

37. Koyanagi, T., Microstrucrual evolution of tungsten exposed to neutron irradiation. To be published, 2016.

38. Fukuda, M., et al., Neutron energy spectrum influence on irradiation hardening and microstructrual development of tungsten. Journal of Nuclear Materials, 2016. 479: p. 249-254. 
39. Setyawan, W., et al., Displacement cascades and defects annealing in tungsten, Part I: Defect database from molecular dynamics simulations. Journal of Nuclear Materials, 2015. 462: p. 329-337.

40. Nandipati, G., et al., Displacement cascades and defect annealing in tungsten, Part III: The sensitivity of cascade annealing in tungsten to the values of kinetic parameters. Journal of Nuclear Materials, 2015. 462: p. 345-353.

41. Faney, T. and B.D. Wirth, Spatially dependent cluster dynamics modeling of microstructure evolution in low energy helium irradiated tungsten. Modelling and Simulation in Materials Science and Engineering, 2014. 22(6): 065010.

42. Marian, J. and T.L. Hoang, Modeling fast neutron irradiation damage accumulation in tungsten. Journal of Nuclear Materials, 2012. 429(1-3): p. 293297.

43. He, J.C., et al., Microstructural development and irradiation hardening of $W$ and W-(3-26) wt\%Re alloys after high-temperature neutron irradiation to $0.15 \mathrm{dpa}$. Nuclear Fusion, 2006. 46(11): p. 877-883.

44. Tanno, T., et al., Effects of Transmutation Elements on Neutron Irradiation Hardening of Tungsten. Materials Transactions, 2007. 48(9): p. 2399-2402.

45. Moteff, J., D.J. Michel, and V.K. Sikka, The influence of irradiation temperature on the hardening behavior of the refractory bcc metals and alloys. Nucl. Metall., 1973. 18: 198211.

46. Rice, P.M. and S.J. Zinkle, Temperature dependence of the radiation damage microstructure in V-4Cr-4Ti neutron irradiated to low dose. Journal of Nuclear Materials, 1998. 258-263: p. 1414-1419.

47. Pokor, C., et al., Irradiation damage in 304 and 316 stainless steels: experimental investigation and modeling. Part II: Irradiation induced hardening. Journal of Nuclear Materials, 2004. 326(1): p. 30-37.

48. Greenwood, L.R. and F.A. Garner, Transmutation of $M o, R e, W, H f$, and $V$ in various irradiation test facilities and STARFIRE. Journal of Nuclear Materials, 1994. 212-215: p. 635-639.

49. Lu, Q., W. Xu, and S.v.d. Zwaag, Designing new corrosion resistant ferritic heat resistant steel based on optimal solid solution strengthening and minimisation of undesirable microstructural components. Computational Materials Science, 2014. 84: p. 198-205. 